\title{
Validation of Existing GNSS Multipath Model
}

\author{
Capucine Amielh, ENAC \\ Alexandre Chabory, ENAC \\ Christophe Macabiau, ENAC \\ Laurent Azoulai, AIRBUS
}

\section{BIOGRAPHY}

Capucine Amielh graduated as an Electronics and Telecommunication engineer in 2015 from ENAC (Ecole Nationale de l'Aviation Civile) in Toulouse, France. Since 2016, she is a PhD student in the Signal Processing and Navigation (SIGNAV) research axis of the TELECOM team at the ENAC. She is working on GNSS multipath model for aircraft surface operations.

\begin{abstract}
Alexandre Chabory graduated in electrical engineering from ENAC (the French Civil Aviation University), Toulouse, France, in 2001. He obtained the Ph.D. degree in electromagnetics from Paul Sabatier University, Toulouse, in 2004. From 2004 to 2007, he was a Post-Doctoral Scientist with the Eindhoven University of Technology. Since 2007, he has been with the Electromagnetics and Antennas Research Group of the ENAC Telecom lab. He is currently the head of this group. His current research interests include electromagnetic theory and modeling, mainly for aeronautical applications.
\end{abstract}

Christophe Macabiau graduated as an electronics engineer in 1992 from the ENAC (Ecole Nationale de l'Aviation Civile) in Toulouse, France. Since 1994, he has been working on the application of satellite navigation techniques to civil aviation. He received his Ph.D in 1997 and has been in charge of the signal processing lab of ENAC since 2000, where he also started dealing with navigation techniques for urban navigation. He is currently the head of the TELECOM lab of ENAC, that includes research groups on signal processing and navigation, electromagnetics and data communication networks.

Laurent Azoulai graduated as an engineer specialized in automatic systems from the Institut Supérieur de l'Electronique de Paris in 1996. He is currently Senior Expert Navigation Systems within Airbus, with 20 years of experience in Navigation engineering applied to Defence and Aviation Systems.

\begin{abstract}
When navigating on the airport surface, aircraft ground position and ground velocity can be estimated using a large variety of sensors, such as Global Navigation Satellite System, Inertial Navigation System, Wheel Speed Sensors and others. The largest contributing sensor is the GNSS, but measurements from this sensor are affected by signal blockage and multipath. In [Che10], a GNSS multipath simulator for airport navigation has been proposed.

In this multipath simulator, up to now, a far-field aircraft+antenna radiation pattern has been used. It has been obtained from measurements at L1 frequency on one scaled aircraft. This means that for different antennas and aircraft, the range errors predicted by the simulator may lose validity. Moreover, the validation of this multipath algorithm has not been fully assessed. The antenna gain pattern used under the multipath simulator is first improved. To do so, a GPS L1 antenna combined with the aircraft structure of the Airbus aircraft family are simulated with an electromagnetic commercial software. For each antenna and aircraft model, the analysis relies on simulations with the electromagnetic software Feko and comparisons. These comparisons show that the different aircraft models have a similar far field gain pattern in accordance with the measurements.

Then we propose a validation of the GPS multipath simulator for GPS L1 C/A signals. This is achieved through the comparison of simulated multipath errors with the multipath errors estimated from real data measurements. The extraction is obtained by means of a Code Minus Carrier (CMC) algorithm coming from a previous AIRBUS/ENAC collaboration in 2012. A number of 52 flights are considered for this validation. By comparing the L1 C/A multipath error variances on a restricted area of Blagnac airport for an elevation bin, we may assess such validation.
\end{abstract}

\section{INTRODUCTION}

Use of aircraft ground position and velocity for aircraft surface navigation is often foreseen to provide operational benefits during surface operations in terms of capacity, efficiency and access. For surface operations, aircraft ground position and ground velocity can be estimated using a large variety of sensors, such as Global Navigation Satellite System, Inertial Navigation System, Wheel Speed Sensors and others. The largest contributing sensor is the GNSS, but measurements from this 
sensor are affected by signal blockage and multipath. In [Chen10], a GPS multipath simulator for airport navigation has been proposed. This model considers interactions with obstacles up to order 2 using Geometrical Optics and Physical Optics methods and accounts for uncertainties in the model parameters, e.g. building positions and materials. This deterministic approach is enhanced by Monte-Carlo simulations. In [Mon14], the multipath simulator has been improved with a multipath ranging error model. This allows to obtain over-bounding laws to get the mean and the variance of bounding distributions on the pseudo-range errors due to multipath.

The intent is now to use the multipath simulator as an input of a more general study, which overall objective is to provide an efficient GNSS multipath error model adequate for aircraft ground position and velocity estimation, including integrity monitoring. This multipath model will provide nominal error, as well as bounds on abnormal pseudo-range error inducing excessive integrity risk for an optimized hybridization algorithm. In our context of ground surface navigation, we define a multiple multipath ranging failure as an abnormally high pseudo-range error due to multipath and occurring simultaneously on several pseudo-range measurements. This model should include consideration of areas close to airport terminals (buildings details, jetways), and the consideration of multiple multipath ranging failures, and mobile obstacles (other aircraft, cars, trucks, buses). The elaborated GNSS multipath model should be applicable to a large variety of airports and aircraft/antenna while using as limited as possible number of inputs. This model should overcome some limitations of the existing model, and therefore should be valid with any ground surface of any nature, not assuming flat ground neither constant height assumption.

In this multipath simulator, up to now, a far-field aircraft plus antenna radiation pattern has been used. It has been measured on one scaled A319 aircraft and antenna at a scaled frequency. This means that when different antennas and aircrafts are considered, the range errors predicted by the simulator may lose validity. Moreover, the validation of this algorithm has been limited to some trajectories on Blagnac airport, France in [Chen10]. In this paper, we only consider L1 C/A signal.

Therefore, the first objective of this paper is to validate the assumption that the antenna plus aircraft can be modeled by a far-field radiation pattern. To do so, a GPS L1 patch antenna combined with the aircraft structure of two types of aircraft will be considered. For each aircraft model, the analysis will rely on simulations with the electromagnetic software Feko.

The second objective of this paper is to propose a validation of the GPS multipath simulator for GPS L1 C/A signals. This will be achieved through the comparison of simulated multipath errors with the multipath errors extracted from real measurements. The extraction is obtained by means of a Code Minus Carrier (CMC) algorithm coming from a previous AIRBUS/ENAC collaboration in 2012. A number of 52 flights involving Blagnac airport are considered for this validation.

\section{AVAILABLE FLIGHT DATA}

Airbus Operations SAS provided ENAC with 106 flight data series. Each flight data set includes reference trajectory and attitude as well as L1 C code, L2 P code, L1 and L2 phase, L1 Signal Noise Ratio and L1 and L2 Doppler measurements from a civil trajectography receiver which is not an MMR. These data contain flight and ground navigation situations. The airports involved are mainly Blagnac airport, France, and Seville airport, Spain. The Table 1 shows the number of available data. In this table, a Blagnac to Blagnac flight accounts for 2 Blagnac ground navigation phases (departure and arrival).

Table 1: Ground navigation phase occurring in the data series

\begin{tabular}{|l|l|l|l|}
\hline Set name & $\begin{array}{l}\text { Number of Blagnac } \\
\text { airport ground } \\
\text { navigation phase }\end{array}$ & $\begin{array}{l}\text { Number of Seville } \\
\text { airport ground } \\
\text { navigation phase }\end{array}$ & $\begin{array}{l}\text { Number of other } \\
\text { airport ground } \\
\text { navigation phase }\end{array}$ \\
\hline W0002 & 51 & 41 & 1 \\
\hline W0003 & 94 & 0 & 2 \\
\hline W0004 & 0 & 14 & 2 \\
\hline
\end{tabular}

In this article, we focus on Blagnac airport, for which a model of the airport has been built up in [Chen10] (Figure 1). This represents 52 trajectories leading to more than 8 million satellite pseudo-range error samples. 

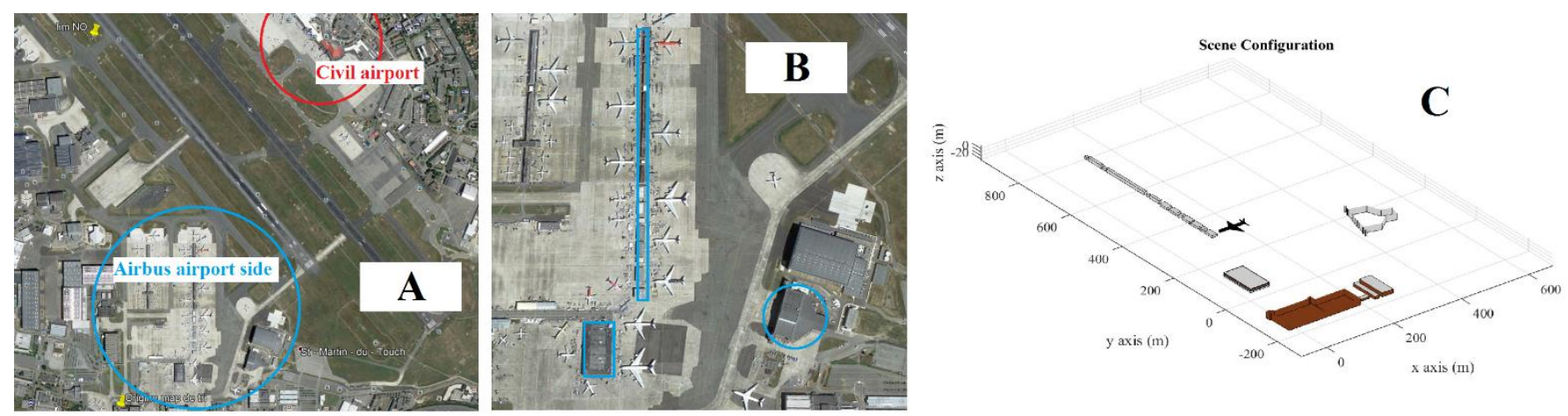

Figure 1: Area of Blagnac airport modeled under the multipath simulator: (A) Blagnac airport,(B) Airbus side of Blagnac airport, (C) Multipath simulator model of the Airbus side of Blagnac airport

The multipath effects depend on the satellite elevation and azimuth, the aircraft velocity, and the aircraft position in the airport environment. Thus, looking at the distribution of the number of data samples for each value of a vector of these parameters is interesting in order to assess whether the dataset is large enough to draw conclusions for any of these parameters. For example, the trajectography receivers had a mask angle of $8^{\circ}$ which prevents us from getting any multipath from lower elevation. The Figure 2 presents the histogram of the number of samples of ground navigation on Blagnac airport per satellite elevation and azimuth. The lower the elevation the higher the number of samples because of the geometry. This is of great interest as multipath propagation has more chance to occur with low elevation satellite.
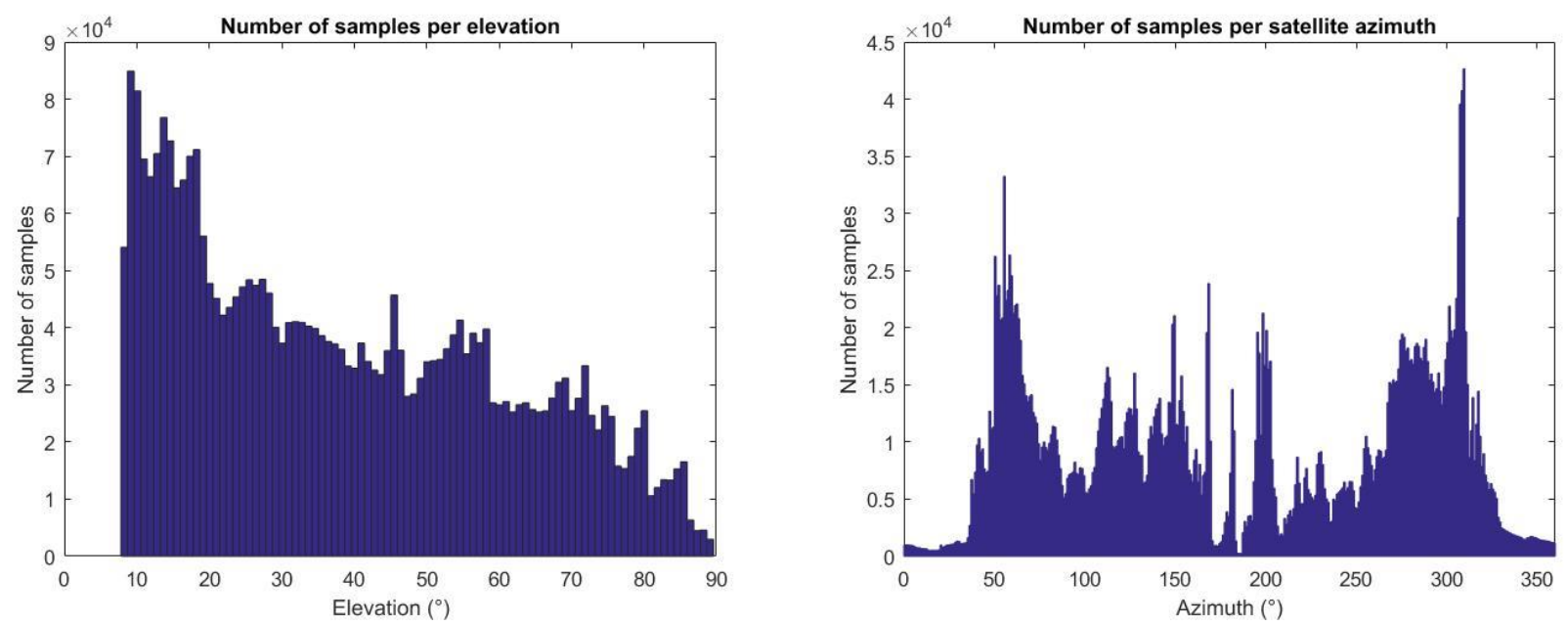

Figure 2: Histogram of the number of samples per elevation and per azimuth 
In terms of space distribution, the ground trajectories are very similar to each other due to the limited area of available taxiways. To represent this distribution, we divided the airport field into $30 \mathrm{~m} \mathrm{x} 30 \mathrm{~m}$ square bins. On Figure 3, we can see the repartition of samples per bin. By chance, we have samples located in the area modeled by the multipath simulator, in around 50 bins.

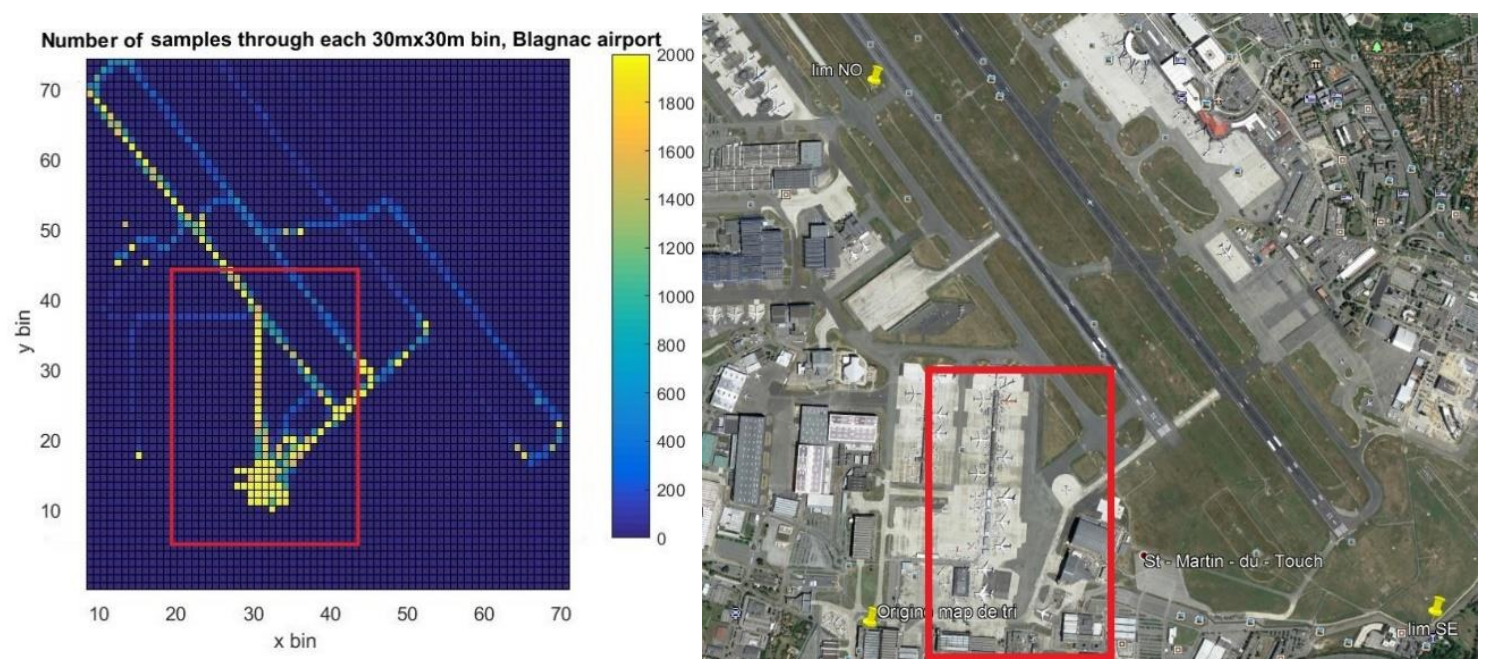

Figure 3: Left: Number of samples through Blagnac airport divided into 30mx30m bins, Right: corresponding area

The histogram of aircraft ground velocity represented in Figure 4Erreur ! Source du renvoi introuvable. correponds to the velocity when the aircraft is located close to the airport area modeled under the multipath simulator.

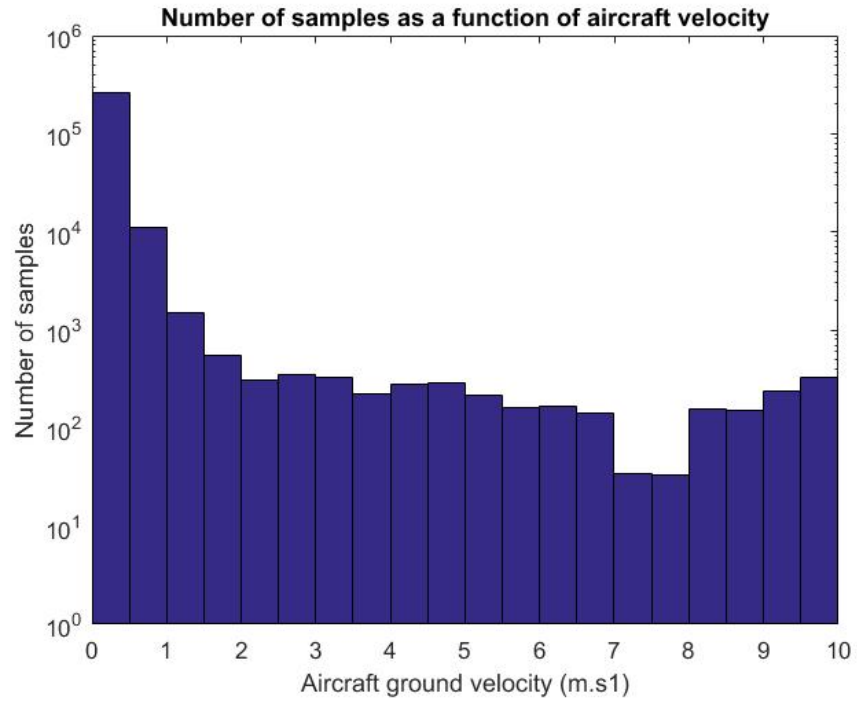

Figure 4: Histogram of the number of samples as a function of aircraft velocity 
These velocities are very low as they correspond to ground navigation on an apron area and gate area (usually between 0 and 20 knots). The phases of surface operations studied through these samples are the stand-lead-in-line taxi, the taxi on taxilane, the taxi on apron taxiway and the taxi on taxiway phases (see Figure 5).

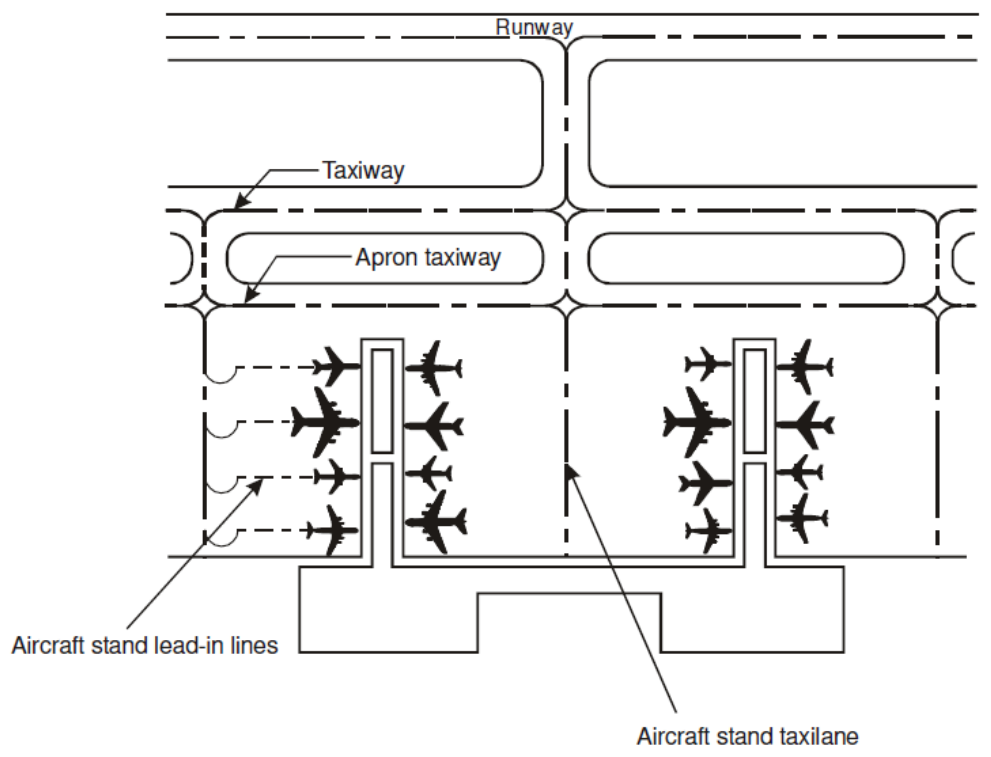

Figure 5: Surface operation areas [ICAO05]

\section{GPS MULTIPATH SIMULATOR FOR AIRCRAFT SURFACE NAVIGATION}

In this section, we present the electromagnetic model of the multipath simulator as well as the multipath pseudo-range error.

\section{A. The electromagnetic multipath model}

The model is based on a deterministic electromagnetic (EM) modeling of the multipath and statistical models of the scene generation. It takes into account 1 st and 2 nd orders interactions (see Figure 6 ) in different ways. The objects in the scene are modeled by means of a polygonal mesh of their surface. The size of each considered object is of at least several wavelengths to ensure the validity of the tangent plane approximation for Physical Optics.
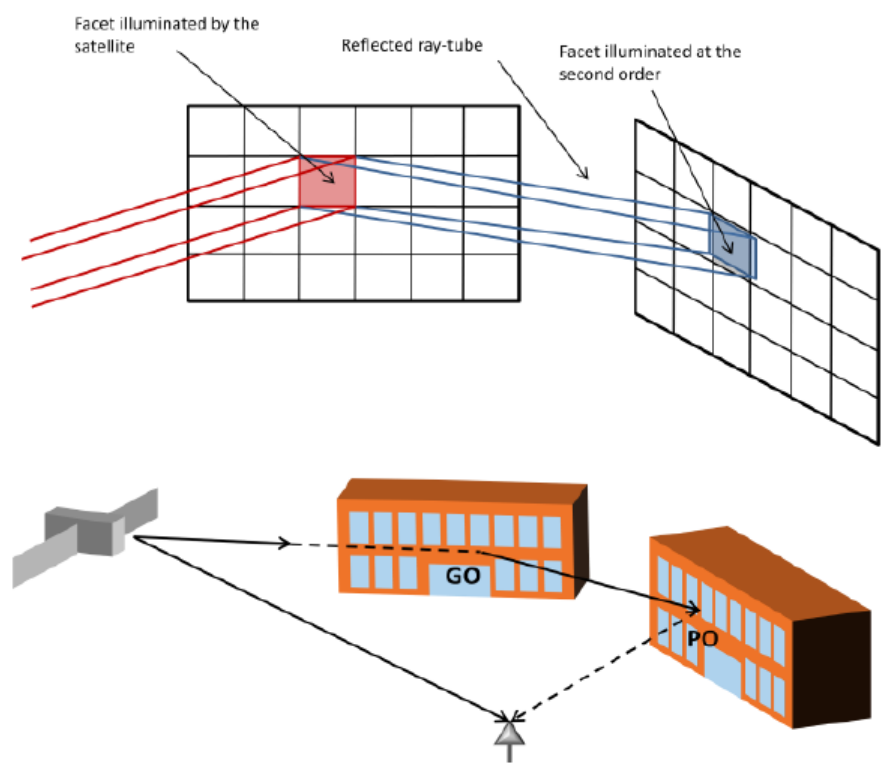

Figure 6: Electromagnetic multipath simulator model 
For $1^{\text {st }}$ order interactions, each illuminated surface generates a scattered field via PO. Each illuminated facet is then the origin of one multipath. The ground reflection is modeled via the image theorem.

For $2^{\text {nd }}$ order interactions, Geometrical Optics (GO) is firstly used to compute the first interaction. GO accounts for the reflection of rays by surfaces. When an incident ray impacts a surface, it yields reflected rays, which direction derives from Snell-Descartes laws, and which amplitude and divergence factor are modified. The divergence factor characterizes the evolution of the field amplitude due to the ray tube divergence with propagation. GO is limited as for instance it predicts a discontinuity of the field at shadow boundaries. The 2 nd interactionis described by a Physical Optics (PO) method. Currents on this 2 nd facet induce an electromagnetic (EM) field by means of the Stratton-Chu integrals. This way, each facet yields one multipath, which characteristics are computed taking into account the antenna effect. This deterministic physical approach is enhanced by MonteCarlo simulations which add a statistical part to the process. Monte-Carlo simulations are performed for transferring model uncertainties inputs into model uncertainties outputs. Here, the uncertainties of the deterministic model are the buildings positions. For several building positions, the EM model is applied. Thus, the mean and the variance of the range errors due to multipath are deduced.

The ENAC model doesn't currently take into account potential mobile obstacles such as other aircraft or mobile jetways, while these 2 types of obstacles seem to have an important influence on the multipath ranging error. The implementation of mobile obstacles and their scattering using PO is left as future work. Moreover, because of the use of PO, small details (windows frame, roughness of surfaces...) are not modeled. The ground is also only modeled using one material, i.e. the distinction between grass and tarmac is not implemented. The validation of the algorithm is also limited to one airport (Blagnac) for some trajectories.

\section{B. The multipath ranging error model}

Montloin et al. [Mon14] have reused the EM deterministic approach developed in [Chen10] for both L1 C/A/L5 bands. The architecture of this process is summarized in Figure 7.

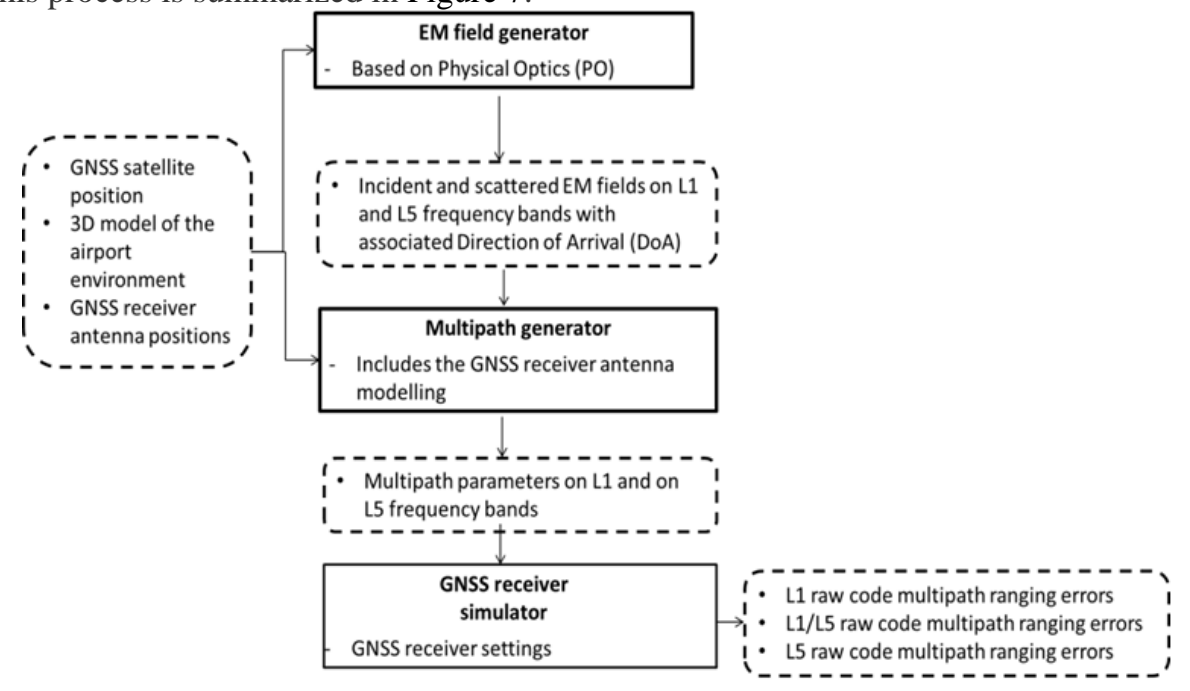

Figure 7: Multipath ranging error model architecture

The EM field generator using GO and PO computations provides the incident and scattered EM field on L1 and L5 frequency bands with their associated Direction of Arrival (DoA). Then, the multipath generator applies the GNSS receiver antenna model to obtain the multipath parameters (delay, amplitude, phase, Doppler) on L1 and L5 bands. The multipath parameters are transferred to the receiver model within the GNSS receiver simulator which computes the raw code multipath ranging errors. The multipath error is then modeled in two distinct situations: the static and the dynamic configurations.

\section{B.1. Static configuration}

In the static configuration, the aircraft (the receiver) and the environment are fixed. The multipath ranging error is then modeled by an over-bounding Gaussian multipath error according to the process in Figure 8.

The impact of multipath is decomposed as an aircraft+ground component and an oscillating component due the scattering of the EM field by the airport obstacles.

Where:

$$
\varepsilon_{m p, L 1 C A+L 5}=b_{A C+\text { ground }}\left(E l, A z_{A C}\right)+b_{o b s}
$$

- $\varepsilon_{m p, L 1 C A+L 5}$, the multipath ranging error, 
- $\quad b_{A C+\text { ground }}\left(E l, A z_{A C}\right)$, the aircraft+ground component induced by the ground reflection, which is considered as a deterministic bias,

- $\quad b_{\text {obs }}$, the oscillating component induced by the $1^{\text {st }}$ and $2^{\text {nd }}$ order interactions with obstacles.

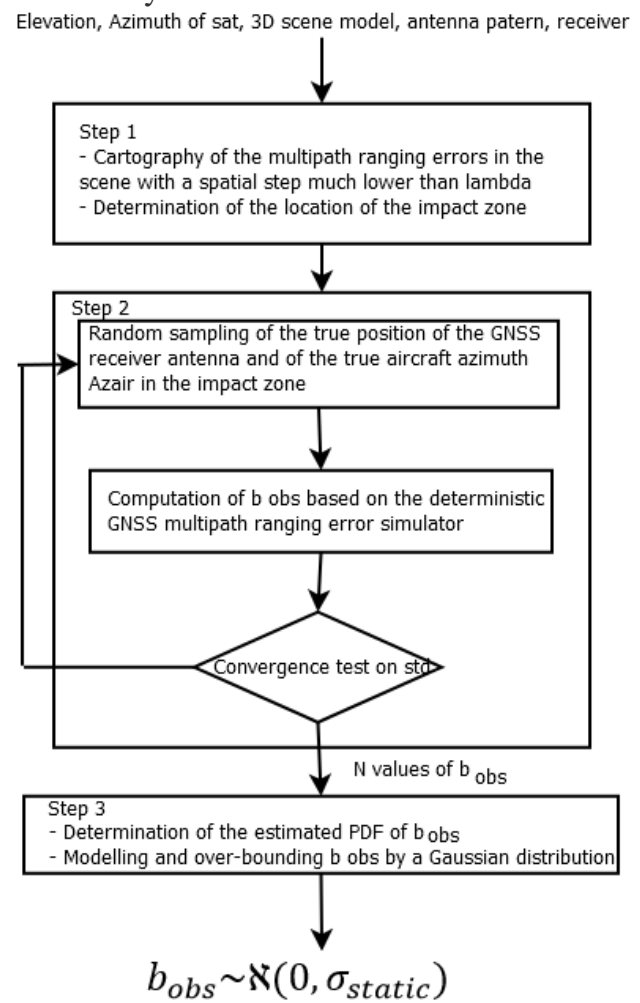

Figure 8: Multipath error model in static configuration

$b_{\text {obs }}$ depends on the GNSS receiver antenna radiation pattern and the GNSS receiver settings, the airborne antenna height, the satellite position, the aircraft azimuth, the aircraft model itself, the $3 \mathrm{D}$ model of the airport environment, and the type of ground material.

We define a static impact zone as a horizontal area of the airport where the magnitude of the code multipath ranging error is significant with respect to other sources of ranging errors (troposphere, ionosphere, satellite clock and ephemeris inaccuracies and receiver thermal noise) in steady state. Then, the Probability Density Function (PDF) of $b_{o b s}$ along the segment considered on the airport surface and the impact zone is estimated by a Monte-Carlo simulation. The inputs of this Monte-Carlo estimation are the aircraft position on the impact zone and the aircraft azimuth angle.

In [Mon14], it has been chosen to over-bound the estimated distribution of $b_{\text {obs }}$ by a Gaussian distribution. Note that this choice has been made as the multipath ranging error model is the basis of an integrity monitoring algorithm. In this algorithm, stochastic measurement error models used to check GNSS measurements consistency and to compute protection levels are overbounding Gaussian distributions. The feasibility to over-bound the estimated PDF by a distribution that best fits the estimated distribution and to design integrity monitoring algorithms that use non-Gaussian distribution as expected measurement error models is left as future work. Moreover, even if the process is supposed to work on other scenarios, the process has only been tested on a limited area of Toulouse Blagnac airport in France.

\section{B.2. Dynamic configuration}

The dynamic configuration process is illustrated in the Figure 9. In this configuration, the ranging multipath error is depicted as Where:

$$
\varepsilon_{m p, L 1 C+L 5}=b_{A C+\text { ground }}\left(E l, A z_{A C}\right)+u_{o b s}(t)
$$

- $\varepsilon_{m p, L 1 C+L 5}$, the multipath ranging error,

- $b_{A C+\text { ground }}\left(E l, A z_{A C}\right)$, the aircraft+ground component induced by the ground reflection which is considered as a deterministic bias, the same as in the static case,

- $u_{o b s}(t)$, the oscillating component induced by the $1^{\text {st }}$ and $2^{\text {nd }}$ order interactions with obstacles, a zero-mean timedependent error. 
$u_{\text {obs }}(t)$ has similar dependency to the GNSS receiver antenna radiation pattern and the GNSS receiver settings, the airborne antenna height, the satellite position, the aircraft azimuth, the aircraft model itself, the 3D model of the airport environment, and the type of ground material considered for $b_{o b s}$ in the static case. But $u_{o b s}(t)$ has also a trajectory location and orientation dependency and an aircraft speed over the trajectory dependency.

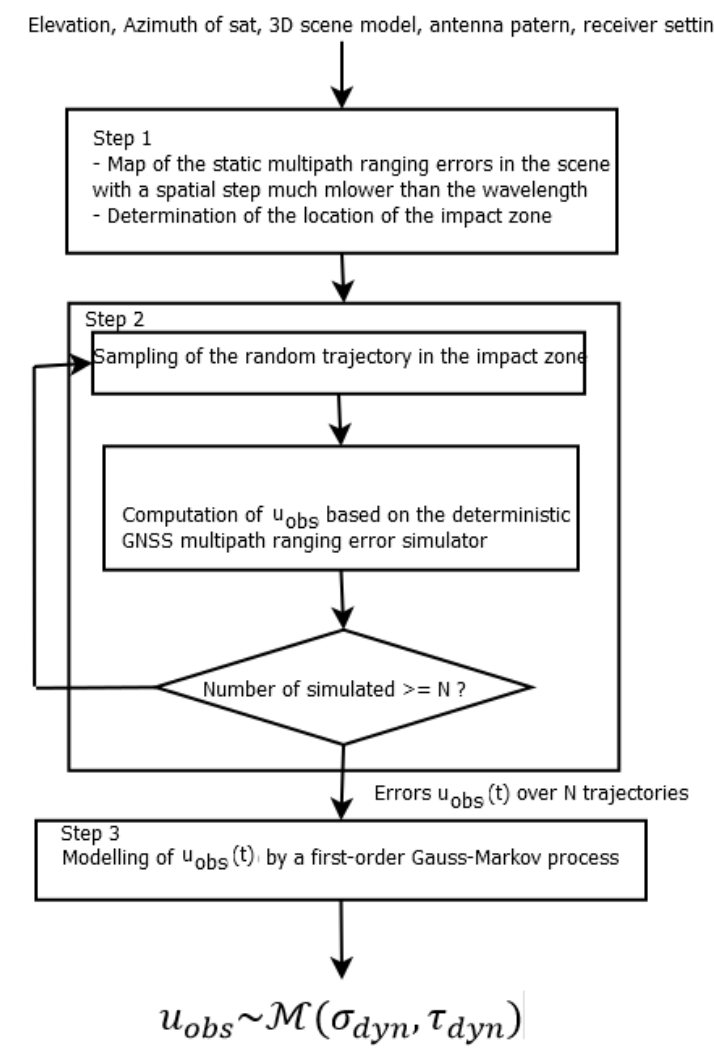

Figure 9: Multipath error model in dynamic configuration

The authors of [Mon14] have provided a stochastic model of the error component $u_{o b s}(t)$ in the impact zone that is independent of the true trajectory of the GNSS receiver antenna in the impact zone. The true trajectory is modeled by its own statistical distribution drawn from Monte-Carlo simulations. In fact, in each Monte-Carlo simulation, each point of a trajectory is randomly chosen within an interval around the true position. This drawn position ensures the true trajectory independency.

In [Mon14] for the dynamic configuration, it has been chosen to over-bound the estimated PSD of $u_{\text {obs }}(t)$ by a zero-mean $1^{\text {st }}$ order Gauss-Markov process $M\left(\tau_{d y n}, \sigma_{d y n}\right)$ where $\tau_{d y n}$ and $\sigma_{d y n}$ are respectively the correlation time and the standard deviation. The normalized PSD of the $1^{\text {st }}$ order Gauss-Markov process is:

$$
P S D_{G M}(f)=\frac{2 \sigma_{d y n}^{2}}{\left(\tau_{d y n}\left((2 \pi f)^{2}+\frac{1}{\tau_{d y n}^{2}}\right)\right)}
$$

To determine $\tau_{d y n}$ and $\sigma_{d y n}$, the authors in[Mon14] have chosen to fix $\tau_{d y n}$ from simulation results and to compute the minimal value of $\sigma_{d y n}$, for which the PSD of the Gauss-Markov process over-bounds the estimated PSD.

We have to emphasize that the algorithm has been tested in a precise scenario and that other conditions may lead to other results (e.g. an aircraft moving between two buildings...). The choice of a Gauss-Markov process has also been made to accommodate the use of an integrity monitoring algorithm.

\section{ANTENNA RADIATION PATTERN}

\section{A. Context}

As mentioned previously, the multipath simulator can use different antenna gain patterns. The reference antenna is a perfectly right handed circularly polarized isotropic antenna. But this model is not very realistic. Therefore, the isotropic L1 gain 
pattern has been improved by an Airbus measurement campaign at a high frequency using scaled A319 aircraft and antenna. The gain has been measured for both right handed and left handed circular polarizations in the horizontal, longitudinal and transversal planes. In [Chen10], an equivalent antenna pattern at L1 has been generated from interpolations of the frequency scaled measurements onto a $5^{\circ} \times 5^{\circ}$ grid in elevation and azimuth. Comparisons between the interpolated and the isotropic gain pattern, demonstrated that the interpolated pattern was more realistic and therefore the best suited.

Nevertheless, in [Chen10], the $5^{\circ} \times 5^{\circ}$ grid in elevation and azimuth led to a smoothed gain pattern which we thought could be improved by simulations. According to the chosen method of simulation, the size of the complete structure of an aircraft may require a too important computation load. Thus, we have to determine the minimal aircraft geometry which yields an acceptable computation time and provides a sufficient accuracy.

According to [Stein04] which performed a measurement campaign on an A340 Airbus family, the main source of multipath is the fuselage. On the other hand, as mentioned in [Chen10], the fuselage can be neglected by assuming that delays induced by multipath are far too low compared to the chip duration to get effect on the antenna. As our multipath simulator should be able to compute pseudo-range errors due to multipath for the whole Airbus aircraft family, one of the purpose of this paper is to determine whether the complete fuselage of each aircraft type has to be considered or not.

\section{B. Antenna mounted on aircraft patterns}

To assess whether the complete fuselage has to be considered or not, we propose two types of simulation geometry: a complete one including a cylindrical fuselage, a conical nose, wings, horizontal and vertical stabilizers with adequate profiles (Fig. A of Figure 10) and a similar one but with a fuselage limited to the surroundings of the antenna of $2 \lambda_{L 1}$ large per $5 \lambda_{L 1}$ long (Fig. B of Figure 10).

We can note that the aircraft itself is a huge structure to mesh for a Method of Moments (MoM) simulation. Therefore, the aircraft is modeled using the Physical Optic method. For higher precision, the GPS patch antenna and its surroundings are kept modeled with MoM in all the simulations. The size of the surrounding area simulated with MoM is a portion of the cylindrical fuselage whose size is $2 \lambda_{L 1}$ large per $5 \lambda_{L 1}$ long.
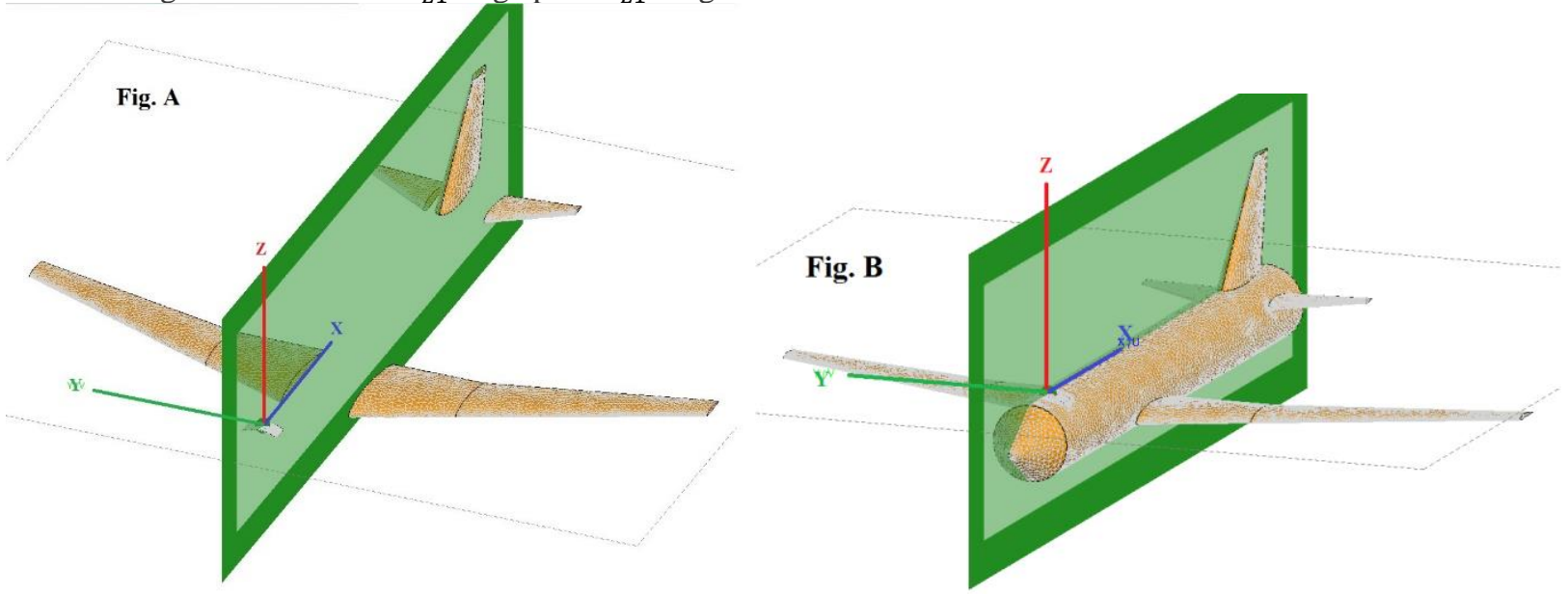

Figure 10: Aircraft geometries used for Feko simulation

In the following, only GPS L1 C/A frequency is considered as the flight data only provides full L1 C/A useful measurements.

Two types of Airbus aircraft are modeled with a GPS L1 patch antenna within Feko. The simulation results for the geometries presented in Figure 10 and the GPS patch antenna are summarized in Figure 11. The aircraft tail is oriented towards the $\mathrm{x}$ axis while the right wing is towards the $\mathrm{y}$ axis. 


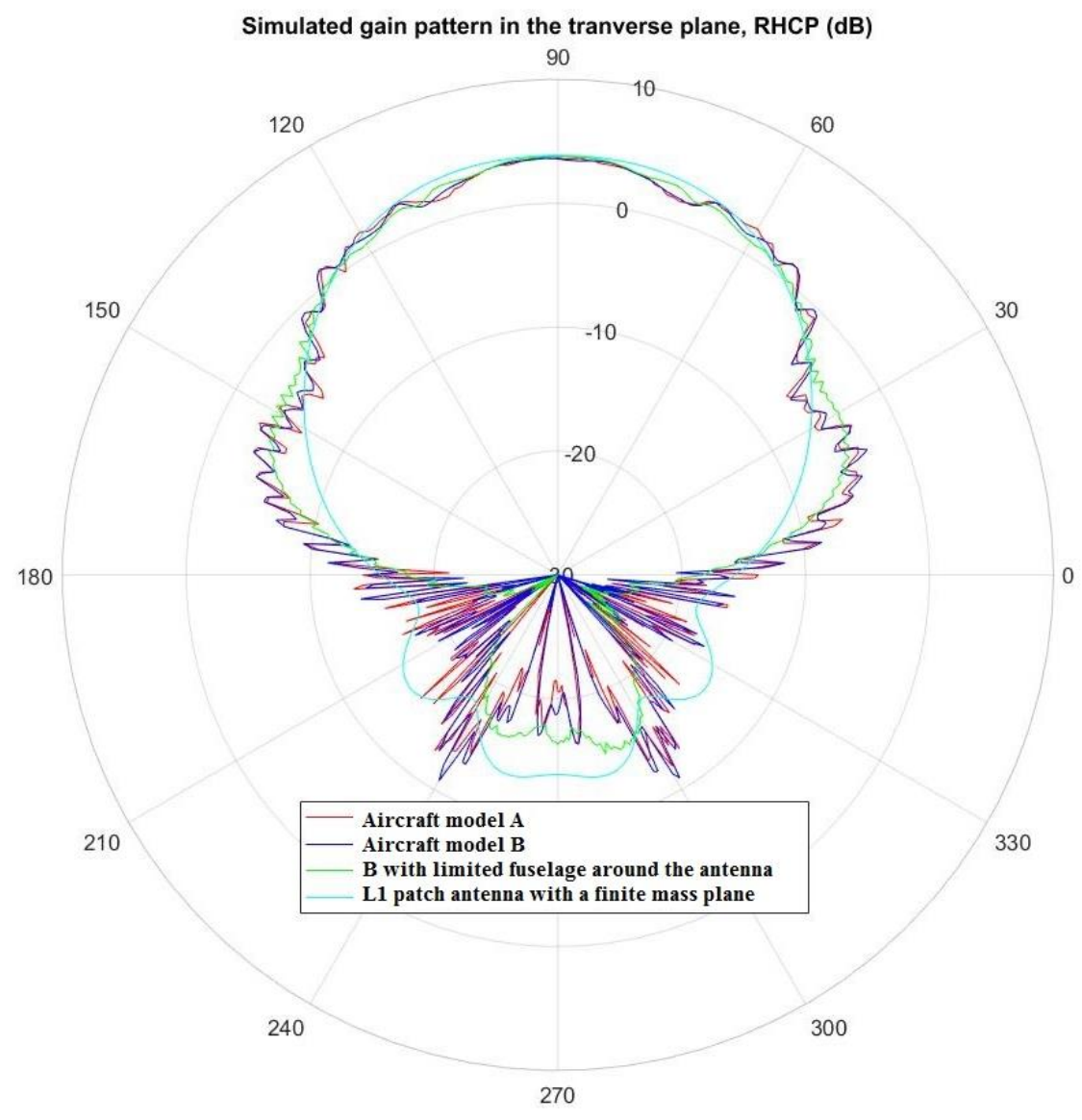

Figure 11: Simulated L1 gain pattern comparison

We can see very similar gain patterns for both Model A and Model B (dark blue and red) aircraft. These results are very interesting as Model A simulation took 10 days to compute the entire system far field and the Model B simulation only 3 days. This is mainly due to the difference of aircraft size. Comparing Model A and Model B patterns, it seems relevant to only consider the Model B pattern in the following. For other Airbus models which are just scaled versions of Model B, it seems also relevant to only consider the Model B radiation pattern.

Then, comparing the green line to the dark blue line, we can see that the small portion of fuselage considered in the Model B is not enough representative of the overall fuselage impact. We can also see that the fuselage structure is the origin of many antenna gain fluctuations. The fact that the closer to the horizontal plane we get, the stronger the gain fluctuations are, confirms, as was described in [Stein04], that the aircraft fuselage is one of the major source of multipath.

To conclude this sub-section, we highlight that the interpolated gain of [Chen10] may be improved by a complete Model B geometry simulation for the whole Airbus aircraft family.

\section{C. Far field assumption}

In [Chen10] and [Mon14], another approximation on antenna pattern has been made: the far field approximation. Until now, the multipath simulator processes signal reflections as if they occur in the far field region of the antenna plus aircraft system. The classical far field zone, the Fraunhoffer region, is determined relatively to the largest dimension of the system $D$ and the wavelength $\lambda$ by $2 D^{2} / \lambda$. For instance, in our context, the aircraft size can be around $50 \mathrm{~m}$ for $\lambda_{L 1}=19 \mathrm{~cm}$. This leads to a theoretical far field region above $26 \mathrm{~km}$. This provides an idea of the problem to model a far field aircraft plus antenna radiation pattern in an environment where obstacles can be only several meters away.

\section{CODE-MINUS-CARRIER EXTRACTION SOFTWARE}

The multipath pseudo-range errors obtained with the multipath simulator are compared to a CMC extraction software. This chapter describes the CMC extraction process.

\section{A. CMC definition}


In our implementation, the Code-Minus-Carrier (CMC) provides a code L1 C/A pseudo-range multipath error estimation by combining L1 and L2 code and carrier measurements. For example, we can express the CMC measurement on L1 C/A band such as:

With:

$$
C M C_{L 1 C A}=C_{L 1}-\lambda_{L 1} \phi_{L 1}-2\left(\lambda_{L 1} \phi_{L 1}-\lambda_{L 2} \phi_{L 2}\right) \cdot \frac{f_{L 2}^{2}}{f_{L 1}^{2}-f_{L 2}^{2}}
$$

- $\quad C_{L 1}$, the code C/A measurement on L1,

- $\quad \lambda_{L 1}$ and $\lambda_{L 2}$, the L1 and L2 wavelengths,

- $\phi_{L 1}$ and $\phi_{L 2}$, the phase measurement on L1 and L2,

- $f_{L 1}=1575.42 \mathrm{MHz}$ and $f_{L 2}=1227.60 \mathrm{MHz}$

The result of equation 3 can be modeled as:

With:

$$
C M C_{L 1 C A} \approx M P_{L 1 C A}+n_{\text {noise }+ \text { track }}+\frac{f_{L 2}^{2}}{f_{L 1}^{2}-f_{L 2}^{2}}\left(\Delta_{L 1}-\Delta_{L 2}\right)-\Delta_{L 1}
$$

- $\quad M P_{L 1 C A}$, the multipath error affecting the L1 C/A code pseudo-range measurement,

- $\quad n_{\text {noise }+ \text { track }}$, the sum of errors due to the receiver L1 C/A dynamics-induced tracking errors and noise, and diffuse multipath,

- $\Delta_{L 1}$ and $\Delta_{L 2}$, the wavelength ambiguity on L1 and L2 in meters,

While the receiver keeps tracking the L1 and L2 carriers of the PRN, the term $\frac{f_{L 2}^{2}}{f_{L 1}^{2}-f_{L 2}^{2}}\left(\Delta_{L 1}-\Delta_{L 2}\right)-\Delta_{L 1}$ remains constant. This way, if we assume that the multipath plus noise error $M P_{L 1 C A}+n_{\text {noise }+ \text { track }}$ should be centered over a sufficient time interval representing a sufficient number of situations, we can remove the bias term $\frac{f_{L 2}^{2}}{f_{L 1}^{2}-f_{L 2}^{2}}\left(\Delta_{L 1}-\Delta_{L 2}\right)-\Delta_{L 1}$ by subtracting the estimated mean value $\overline{C M C_{L 1 C A}}$ of the CMC measurement $C M C_{L 1 C A}$ between two instants of loss tracking. At this stage, the algorithm provides an estimate $\left\langle M P_{L 1 C}(k)+n_{\text {noise }+ \text { track }_{L 1}}\right\rangle$ of the multipath plus noise error affecting the L1 C/A code pseudo-range measurement.

$$
\left\langle M P_{L 1 C A}(k)+n_{n o i s e+\operatorname{track}_{L 1}}\right\rangle(k)=C_{L 1}(k)-\lambda_{L 1} \phi_{L 1}(k)-2\left(\lambda_{L 1} \phi_{L 1}(k)-\lambda_{L 2} \phi_{L 2}(k)\right) \cdot \frac{f_{L 2}^{2}}{f_{L 1}^{2}-f_{L 2}^{2}}-\overline{C M C_{L 1 C A}}
$$

As the flight data does not include any L1 P neither L2 C measurements, the only other CMC we may obtain is the L2 P one.

$$
\left\langle M P_{L 2 P}(k)+n_{\text {noise }+\operatorname{track}_{P 2}}\right\rangle(k)=P_{L 2}(k)-\lambda_{L 1} \phi_{L 1}(k)-2\left(\lambda_{L 1} \phi_{L 1}(k)-\lambda_{L 2} \phi_{L 2}(k)\right) \cdot \frac{f_{L 2}^{2}}{f_{L 1}^{2}-f_{L 2}^{2}}-\overline{C M C_{L 1 C A}}
$$

It is however interesting to notice that the term $C M C_{L 1 C A}$ in reality also reflects some code tracking biases due to satellite and user antenna patterns, plus nominal signal deformation error, as well as phase tracking errors due to phase center variations, as shown in [Wan17]. At this stage of our analysis, these biases will be reflected in the estimated multipath error, but will not be separately estimated. Other investigations are ongoing in different organizations and in our lab on this topic.

\section{B. Estimation of multipath error statistic}

\section{B.1. Multipath plus noise error variance estimate}

By means of the CMC extraction process, we get an estimation of the multipath plus noise errors. These MP + noise estimates are time-correlated due to the processing in the DLL, but no further correlation is applied as the recorded pseudo-range measurements are unsmoothed. Before estimating the variance of the $M P+$ noise observations, the measurements are sorted to define a ground data set and a flight data set. These sets are then sorted by $1^{\circ}$ elevation bins. Thus, we can express the estimated variance of the multipath plus noise error on the $j^{\text {th }}$ elevation bin for L1 C, $\hat{\sigma}_{M P+n o i s e_{L 1 C A}}^{2}(j)$.

Where

$$
\hat{\sigma}_{M P+\text { noise }_{L 1 C}}^{2}(j)=\frac{1}{n_{j}-1} \sum_{i=1}^{n_{j}}\left(x_{j}(i)-\overline{x_{J}}\right)^{2}
$$

- $n_{j}$ is the number of independent samples in the $j^{\text {th }}$ bin,

- $\quad x_{j}(i)$, the $j^{\text {th }}$ multipath plus noise error affecting the L1 C/A code pseudo-range measurement, $x_{j}=$ $\left\langle M P_{L 1 C A}(k)+n_{\text {noise }+ \text { track }_{L 1}}\right\rangle_{\text {elevbinj }}$,

- $\bar{x}_{j}$, the mean value of $x_{j}$.

According to [Papou91], $\hat{\sigma}_{M P+\text { noise }_{L 1 C A}}^{2}(j)$ is an unbiased and consistent estimate of $\sigma_{M P+\text { noise }_{L 1 C A}}^{2}(j)$. 


\section{B.2. Multipath variance estimate}

Assuming that the multipath component and the noise plus dynamics receiver tracking error component are statistically independent, we may write the estimated variance of the multipath plus noise error as the sum of the multipath error variance estimate and the receiver noise plus dynamics tracking error variance estimate.

With

$$
\hat{\sigma}_{M P+n o i s e_{L 1 C A}}^{2}(j)=\hat{\sigma}_{M P_{L 1 C A}}^{C M C}{ }^{2}(j)+\hat{\sigma}_{\text {track }_{L 1 C A}}^{2}(j)
$$

- $\hat{\sigma}_{M P_{L 1 C A}}^{2}$, the multipath error variance estimate in the $j^{\text {th }}$ bin and,

- $\hat{\sigma}_{\text {track }_{L 1 C A}^{2}}^{2}(j)$, the receiver noise plus dynamics tracking error variance estimate in the same bin.

We then can express an estimate of the multipath error standard deviation, $\hat{\sigma}_{M P_{L 1 C A}}^{C M C}(j)$ :

$$
\hat{\sigma}_{M P_{L 1 C A}}^{C M C}(j)=\sqrt{\hat{\sigma}_{M P+n o i s e_{L 1 C A}}^{2}(j)-\hat{\sigma}_{\text {track }_{L 1 C A}^{2}}(j)}
$$

The receiver tracking error variance model as a function of the elevation angle, $\hat{\sigma}_{\text {track }_{L 1 C A}}(j)$, is determined using a method similar to that in [Boo00]. In fact, by combining a $C / N 0$ as a function of the elevation model to receiver noise model also a function of the elevation, we can get an estimation of the receiver noise as a function of the elevation.

\section{VALIDATION METHODOLOGY FOR MULTIPATH ERROR VARIANCE MODEL}

After describing how the L1 C/A multipath pseudo-range error variance is obtained in the CMC software, we have to establish an equivalent definition for the multipath simulator to compare these two variances. This section presents how the comparison is performed.

\section{A. Multipath error variance model of the multipath simulator}

As the receiver noise model provided by the CMC extraction software is related to the elevation, we only present the influence of the elevation and the location on the map on the multipath pseudo-range error variance.

The airport field may be divided into square bins. Within each bin, we can gather the samples per elevation. Assuming we have enough samples into the elevation sub-bin, by defining statistical trajectories around the recorded one passing through the same bin, we can obtain a multipath error variance prediction per space bin per elevation sub-bin.

Where

$$
\hat{\sigma}_{M P_{L 1 C A}}^{2}(k, j)=\frac{1}{n_{k j}-1} \sum_{i=1}^{n_{k j}}\left(y_{k j}(i)-y_{k J}^{\prime}\right)^{2}
$$

- $n_{k j}$ is the number of independent samples in the $k^{t h}$ space bin and $j^{t h}$ elevation bin,

- $\quad y_{k j}(i)$, the $i^{t h}$ simulated multipath error affecting the L1 C/A code pseudo-range in the $k^{\text {th }}$ space bin and $j^{\text {th }}$ elevation bin,

- $y_{k J}^{\prime}$, the mean value of $y_{k j}$.

The standard deviation of the simulated multipath error $\hat{\sigma}_{M P_{L 1 C}}(k, j)$, in the $k^{\text {th }}$ space bin and $j^{\text {th }}$ elevation bin, is then

\section{B. Multipath error variance test}

$$
\hat{\sigma}_{M P_{L 1 C A}}(k, j)=\sqrt{\hat{\sigma}_{M P_{L 1 C A}}^{2}(k, j)}
$$

To decide whether the multipath simulator is wrong or not, we perform a $\chi^{2}$ test on the simulated variance with regard to the CMC variance. The test hypothesis $H_{0}$ and $H_{1}$ are the following

$$
\begin{gathered}
H_{0}: \hat{\sigma}_{M P_{L 1 C A}}^{2}(k, j)=\hat{\sigma}_{M P_{L 1 C A}}^{C M C}{ }^{2}(k, j) \\
H_{1}: \hat{\sigma}_{M P_{L 1 C}}^{2}(k, j) \neq \hat{\sigma}_{M P_{L 1 C}}^{C M C}{ }^{2}(k, j)
\end{gathered}
$$

Where $\hat{\sigma}_{M P C 1 C}^{C M C}{ }^{2}(k, j)$ is the variance of the pseudo-range error due to multipath on L1 C/A on the $k^{\text {th }}$ space bin and $j^{t h}$ elevation bin and is equal to

With

$$
\hat{\sigma}_{M P L 1 C}^{C M C}{ }^{2}(k, j)=\hat{\sigma}_{M P+\text { noise }_{L 1 C}}^{C M C}(k, j)-{\hat{t_{\text {track }}}}_{L 1 C}^{\text {CMC }}{ }^{2}(j)
$$

- $\quad \sigma_{M P+\text { noise }}^{C M C}{ }^{2}(k, j)$, the multipath plus noise pseudo-range variance on the $k^{\text {th }}$ space bin and $j^{\text {th }}$ elevation bin, and

- $\quad_{\text {track }_{L 1 C}}^{C M C}(j)$, the receiver noise plus dynamics tracking error variance on the overall CMC measurements at the same elevation than the $j^{t h}$ elevation bin. This quantity should be noted $\hat{\sigma}_{\operatorname{track}_{L 1 C}{ }^{C M C}}{ }^{2}(E l(j))$ but for simplicity we will keep the previous notation.

The test statistic used is the usual $\chi_{S T A T}^{2}$ following a chi-square distribution with N-1 degree of freedom.

Where

$$
\chi_{S T A T}^{2}=\frac{(N-1) S^{2}}{\sigma^{2}}
$$


- $\quad N$ is the number of samples,

- $S^{2}$ is the sample variance, and

- $\sigma^{2}$ is the hypothesized population variance.

Which can be written in our case as $\chi_{S T A T}^{2}=\frac{\left(n_{k j}-1\right) \theta_{M P_{L 1 C}}^{2}(k, j)}{{\sigma_{M P C 1 C}^{C M C}{ }^{2}(k, j)}^{2}}$

For a chosen level of significance $\alpha$, we will reject $H_{0}$ if $\chi_{S T A T}^{2}<\chi_{\alpha \backslash 2}^{2}$ or if $\chi_{S T A T}^{2}>\chi_{1-\alpha \backslash 2}^{2}$.

These types of multipath pseudo-range error variance tests could be performed for the multipath plus noise variance described as a function of other parameters and not just elevation parameter. Aircraft velocity and attitude, as well as satellite orientation relative to the obstacles have a great influence on multipath. The estimation of such related multipath error variance is limited by the noise and dynamics tracking error variance estimation model. One could divide the space into bins, themselves divided into velocity bin, for instance.

\section{METHODOLOGY FOR FAR FIELD ASSUMPTION VALIDATION}

We highlighted in 3.C that the size of an antenna+aircraft radiating system induces a far field limit larger than the distance to obstacles. Therefore, the far field assumption used to simulate the interaction between a close obstacle and the receiver is uncertain.

If we consider an antenna-only radiating system, the far field limit is smaller than the minimal distance to an obstacle. Thus, in this case, the far field assumption is correct.

For instance, the size of our patch antenna is $\lambda_{L 1}$ which lead to a far field limit of $2 \lambda_{L 1}=38 \mathrm{~cm}$. Consequently, to know if the far field assumption, on the aircraft plus antenna system, is correct in our aircraft ground navigation context, one could compare in the multipath simulator:

- A configuration which includes the antenna-only radiation pattern mounted on an aircraft model (Fig. B of Figure 12) close to an obstacle,

- Another configuration that includes the A320 radiation pattern presented in Section 3.B close to the same obstacle (Fig. A Figure 12).

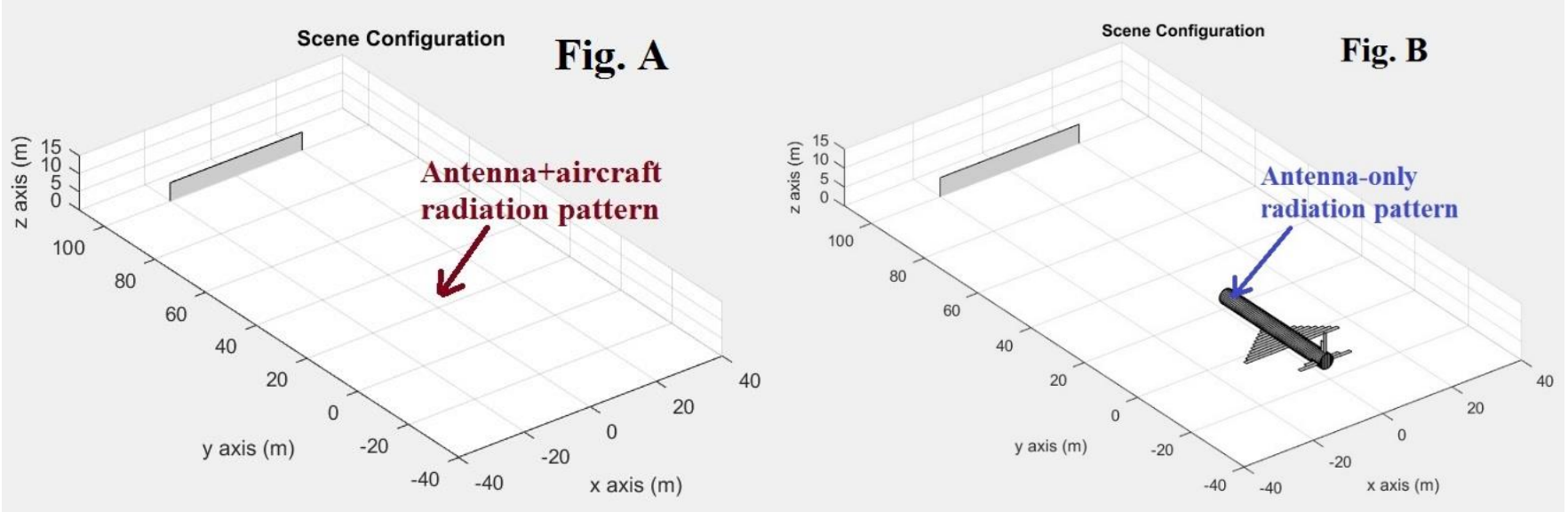

Figure 12: Scene configuration for far-field validation: Left: Antenna-aircraft Feko pattern as input, Right: Antenna-only Feko pattern as input

If both configurations result in similar multipath pseudo-range errors, we can positively conclude on the validity of the far field assumption as well as on the correct multipath simulator aircraft model.

The application of this methodology will be presented in another paper.

\section{VALIDATION}

\section{A CMC measurements}

First of all, we compute the multipath plus noise error using the CMC software on the complete dataset. This allows us to have a reliable receiver noise plus dynamics tracking error variance per elevation bin as we have many elevation samples. Figure 13 shows the L1 C/A multipath plus noise pseudo-range error variance per 30mx30m ground plane bin on the whole Blagnac airport across all elevation, azimuth and velocity. It is assumed that multipath and noise errors are stationary with each $30 \mathrm{mx} 30 \mathrm{~m}$ ground plane bin. We can identify the bins where multipath amplitudes are the strongest. 


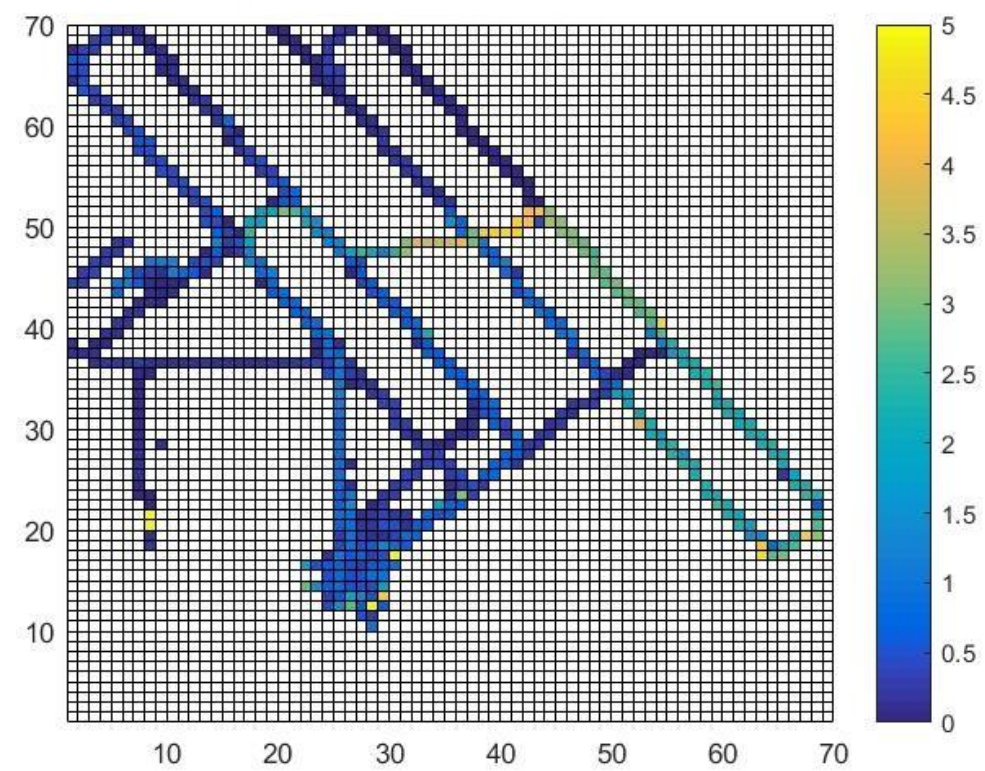

Figure 13: L1 C/A multipath plus noise pseudo-range error variance from the CMC software

The multipath plus noise pseudo-range error for the ground samples located on Blagnac airport can be seen in Figure 14. On the quantile-quantile plot of the Figure 14, the distribution is likely Gaussian around 0 until one sigma probably due to the noise. We can also see the influence of the multipath in higher values of multipath plus noise pseudo-range error, which leads to a non-Gaussian distribution as soon as the error is larger than its standard deviation.
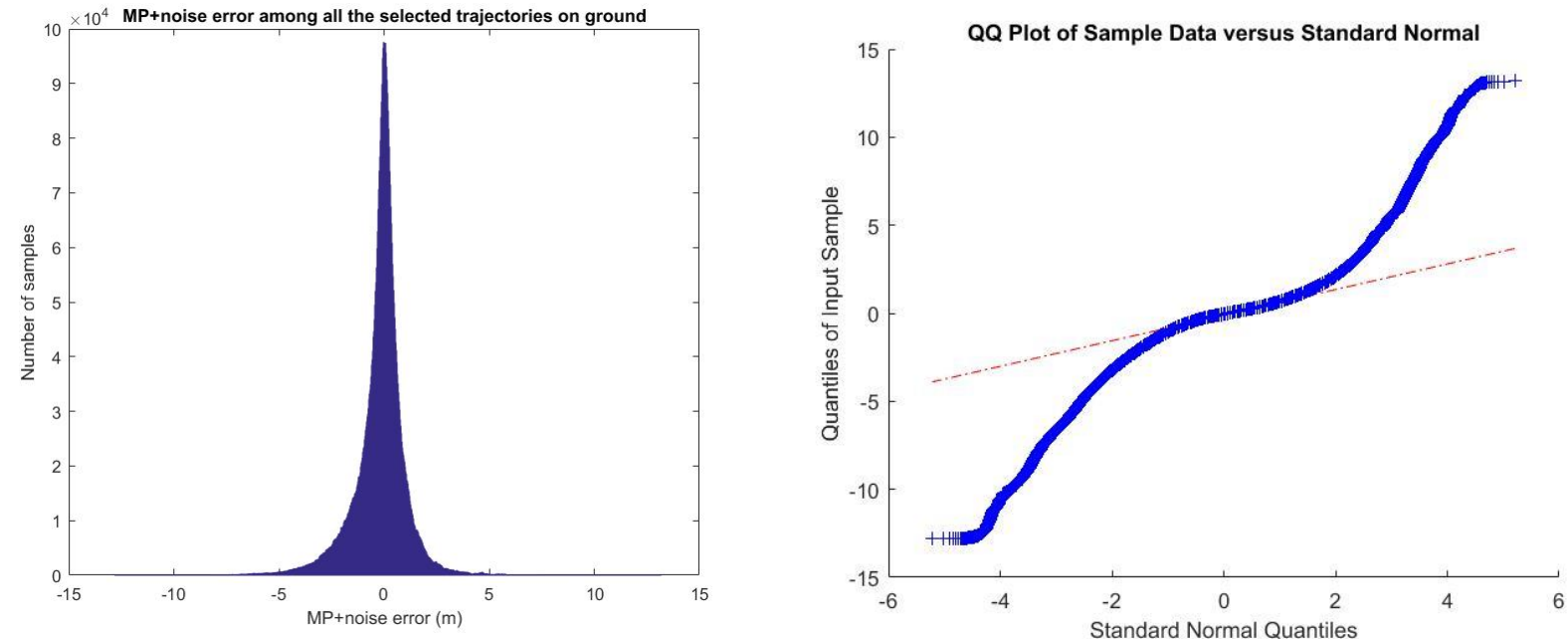

Figure 14: Multipath plus noise pseudo-range error for the selected samples

\section{B. Simulation and measurement comparison of time series}

For each simulation, the multipath simulator draws several parameters: the building positions in the horizontal plane, the building orientations in the horizontal plane, the building height, the building materials, the building thicknesses, the ground material, the scene altitude and the receiver position. At each point of a trajectory, the multipath simulator has to recalculate all the multipath parameters by computing the reflections of all the illuminated facets, and this represents typically on this scene between 1000 and 2000 terms. This leads to a long computation time. Therefore, rather than computing a whole trajectory, we simulate portions representing typical situations. The following figures present the pseudo-range time series in different static and dynamic cases.

Figure 15 illustrates the errors obtained when the aircraft is waiting at the stand for two satellites at low elevation. It's in this situation that the aircraft is the closest to the building. 

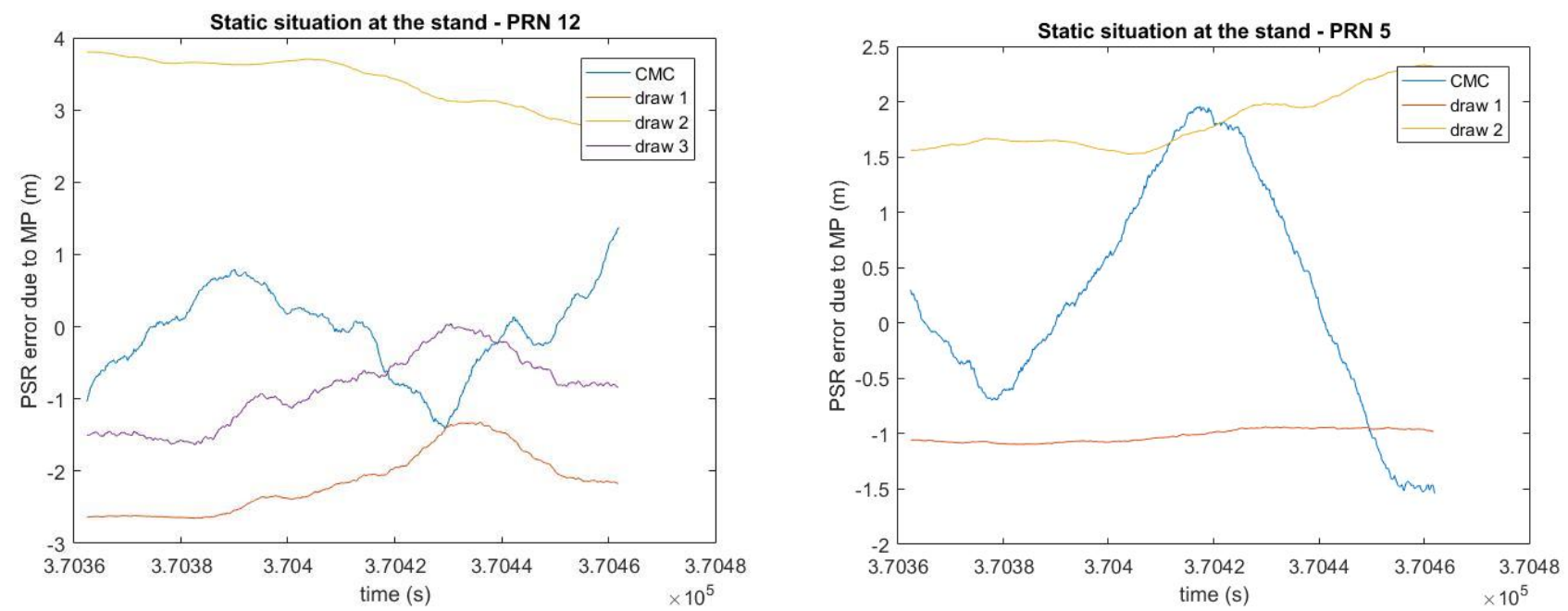

Figure 15: Pseudo-range error due to multipath in a static configuration on one trajectory, waiting at the stand, PRNs 12 and 5

\begin{tabular}{|l|l|l|l|}
\hline Static - PRN 12 & Area & Mean $(\mathrm{m})$ & Variance $(\mathrm{m})$ \\
\hline CMC & At the stand & -0.063 & 0.583 \\
\hline Draw 1 & At the stand & -2.147 & 0.422 \\
\hline Draw 2 & At the stand & 3.392 & 0.340 \\
\hline Draw 3 & At the stand & -0.865 & 0.497 \\
\hline Static - PRN 5 & Area & Mean $(\mathrm{m})$ & Variance $(\mathrm{m})$ \\
\hline CMC & At the stand & 0.257 & 1.010 \\
\hline Draw 1 & At the stand & -1.017 & 0.058 \\
\hline Draw 2 & At the stand & 1.810 & 0.249 \\
\hline
\end{tabular}

Table 1: Statistics of the static case at the stand

In the case of a static configuration of 100 seconds, we may expect few variations of the multipath pseudo-range error. This is what we observe in simulation drawings which present low variances. But the pseudo-range error variance of CMC measurements observed for the PRN 5 is above $1 \mathrm{~m}$. This may be explained by the presence of aircraft in the scene which influence the CMC but are not taken into account in our simulations as demonstrated in [Chen10]. But tjis may not be the only reason.

Figure 16 presents CMC measurements and simulations time series in a situation where the aircraft has been pushed back from the stand and is waiting to taxi by its own power.
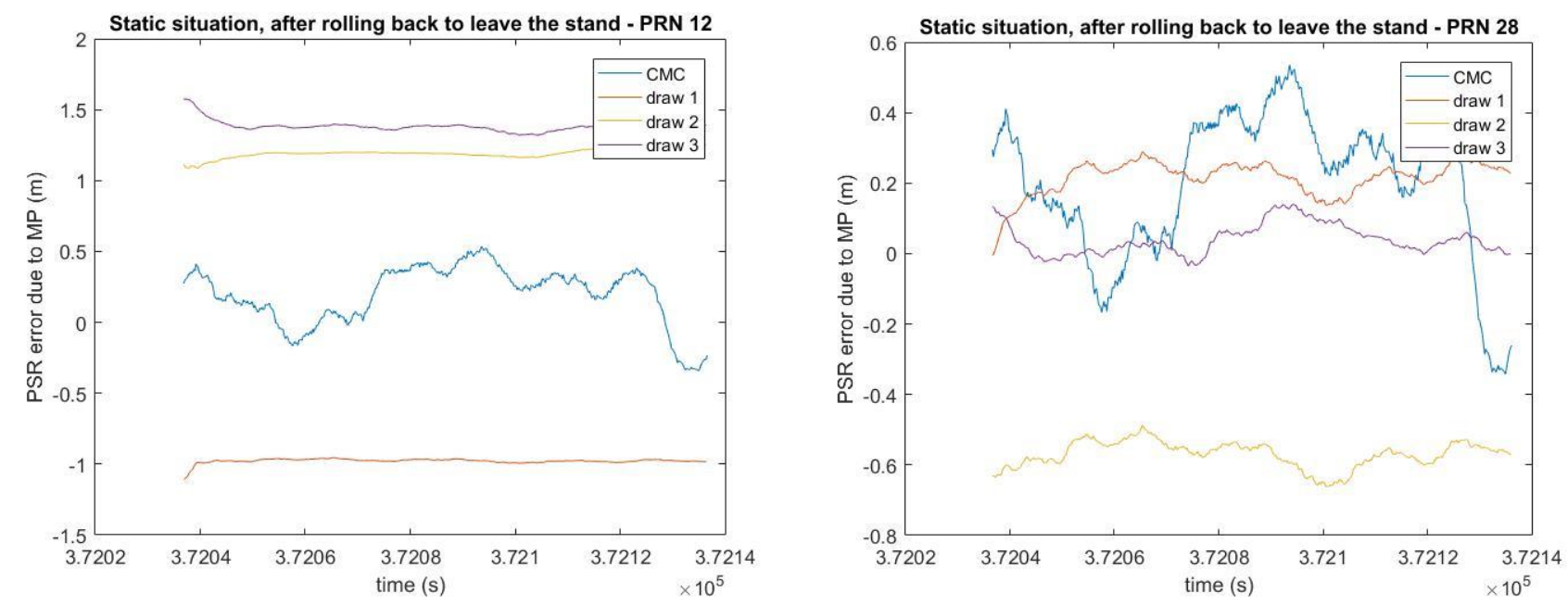

Figure 16: Pseudo-range error due to multipath in a static configuration on one trajectory, waiting after push back, PRNs 12 and 28 


\begin{tabular}{|l|l|l|l|}
\hline Static - PRN 12 & Area & Mean $(\mathrm{m})$ & Variance $(\mathrm{m})$ \\
\hline CMC & After push back & 0.197 & 0.205 \\
\hline Draw 1 & After push back & 0.214 & 0.037 \\
\hline Draw 2 & After push back & -0.570 & 0.033 \\
\hline Draw 3 & After push back & 0.040 & 0.043 \\
\hline Static - PRN 28 & Area & Mean $(\mathrm{m})$ & Variance $(\mathrm{m})$ \\
\hline CMC & After push back & 0.195 & 0.207 \\
\hline Draw 1 & After push back & -0.978 & 0.017 \\
\hline Draw 2 & After push back & 1.930 & 0.033 \\
\hline Draw 3 & After push back & 1.384 & 0.039 \\
\hline
\end{tabular}

Table 2: Statistics of the static case after rolling back to leave the stand

We get results very similar to the previous figure with lower variances in simulation than in the measurements. We can note that the mean of each simulation is very different from one another. This is explained by the phase variation induced by the drawn uncertainties.

The following figures, Figure 17 and Figure 18, illustrate different dynamic situations. In Figure 17, the aircraft is pushed back by a truck to leave the stand while in Figure 18 the aircraft is performing taxi on the apron parallel to the stands to join the taxiway.
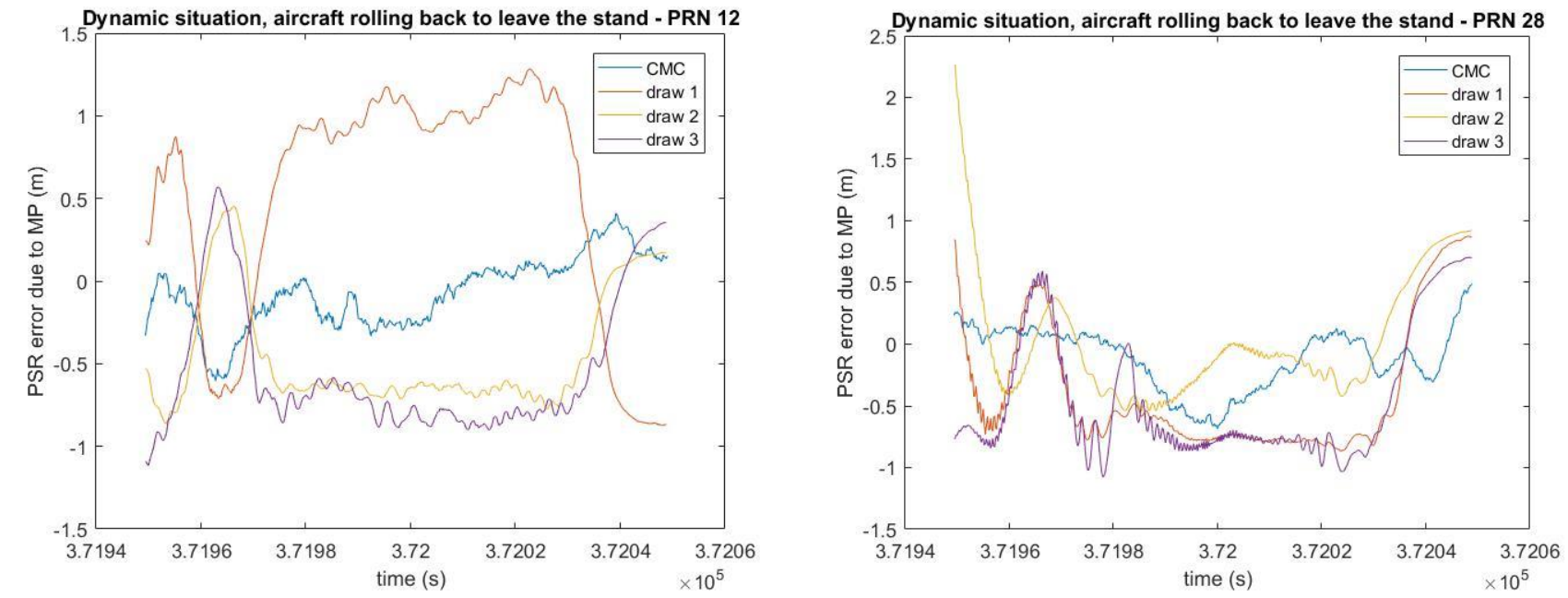

Figure 17: Pseudo-range error due to multipath in a dynamic configuration on one trajectory, push back, PRNs 12 and 28

\begin{tabular}{|l|l|l|l|}
\hline Dynamic - PRN 12 & Area & Mean $(\mathrm{m})$ & Variance $(\mathrm{m})$ \\
\hline CMC & Pushed back & -0.069 & 0.216 \\
\hline Draw 1 & Pushed back & 0.508 & 0.707 \\
\hline Draw 2 & Pushed back & -0.449 & 0.356 \\
\hline Draw 3 & Pushed back & -0.544 & 0.417 \\
\hline Dynamic - PRN 28 & Area & Mean $(\mathrm{m})$ & Variance $(\mathrm{m})$ \\
\hline CMC & Pushed back & -0.113 & 0.256 \\
\hline Draw 1 & Pushed back & -0.384 & 0.530 \\
\hline Draw 2 & Pushed back & 0.039 & 0.511 \\
\hline Draw 3 & Pushed back & -0.457 & 0.515 \\
\hline
\end{tabular}

Table 3: Statistics of the dynamic case: aircraft rolling back to leave the stand 

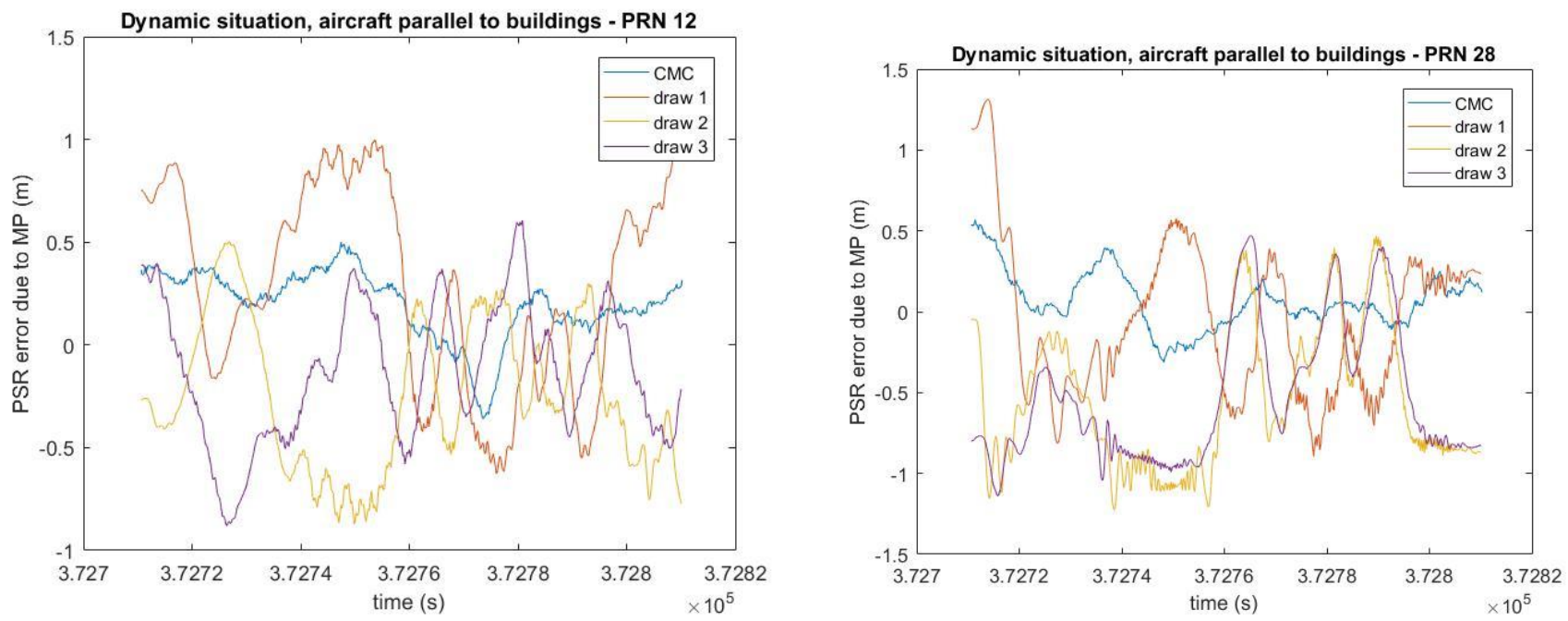

Figure 18: Pseudo-range error due to multipath in a dynamic situation, roll parallel to the stands, PRN 12 and 28

\begin{tabular}{|l|l|l|l|}
\hline Dynamic - PRN 12 & Area & Mean $(\mathrm{m})$ & Variance $(\mathrm{m})$ \\
\hline CMC & Parallel to stands & 0.199 & 0.167 \\
\hline Draw 1 & Parallel to stands & 0.288 & 0.485 \\
\hline Draw 2 & Parallel to stands & 0.243 & 0.363 \\
\hline Draw 3 & Parallel to stands & -0.131 & 0.333 \\
\hline Dynamic - PRN 28 & Area & Mean $(\mathrm{m})$ & Variance $(\mathrm{m})$ \\
\hline CMC & Parallel to stands & 0.068 & 0.175 \\
\hline Draw 1 & Parallel to stands & -0.053 & 0.477 \\
\hline Draw 2 & Parallel to stands & -0.536 & 0.430 \\
\hline Draw 3 & Parallel to stands & -0.532 & 0.418 \\
\hline
\end{tabular}

Table 4: Statistics of the dynamic case: roll parallel to the stands

In these 4 dynamic cases, we may note a convergence phase on the first instant due to the fact that the correlator outputs are directly placed in a dynamic situation where multipath occurs while the carrier and code tracking loops local replicas do not assume the presence of multipath. Nevertheless, on the following instants, after the convergence, the pseudo-range error magnitudes are similar. The estimated means are sometimes very close to each other. Also, we can note that the variances of the simulated data are very similar to each other and are higher than the measured one (at least doubled). This is partly due to the convergence period where the multipath pseudo-range errors are high.

In Figure 18, the aircraft is moving parallel to the stands. This implies that the receiver is affected by the same antenna bias and aircraft multipath all along. Moreover, the aircraft velocity is higher during the taxi phase than during the push-back and Montloin et al. [Mon14] have shown that the aircraft velocity is a possible source of lower variances. This may explain why the variance during the push-back is higher than during this taxi phase.

\section{CONCLUSION}

In this paper, we have exposed a proposed validation methodology of the L1 C/A pseudo-range error variance due to multipath. Our study relies on flight data measured on Blagnac airport, provided by Airbus. We have extracted the multipath plus noise pseudo-range errors using a CMC extraction software. This same software permitted to get rid of the receiver noise plus dynamic tracking error variance as a function of the satellite elevation.

By dividing Blagnac airport into 30mx30m square bin over which we assume that the multipath plus noise error is stationary, we have been able to analyze the multipath pseudo-range errors on reduced size situations. In the future, our intent is to compare the L1 C/A multipath pseudo-range error variance from the CMC software and the one from the multipath simulator by means of a $\chi^{2}$ variance test.

However, in this paper, to analyze the driving parameters and to reduce the computation time, we have only compared pseudo-range error time series on different $30 \mathrm{mx} 30 \mathrm{~m}$ ground bins corresponding to representative situations. Comparing CMC pseudo-range error time series affected by noise to the equivalent time series of the multipath simulator is complicated as tens of Monte-Carlo draws (taking into account variations of the building positions in the horizontal plane, the building orientations 
in the horizontal plane, the building height, the building materials, the building thicknesses, the ground material, the scene altitude and the receiver position) should be performed to get trustful error statistics. Nevertheless, with only 3 draws, we can see that the simulated variances are very close to each other. A study of the variance convergence in dynamic configurations is ongoing at ENAC.

An initial convergence time period appeared on simulated pseudo-range errors on portions of trajectory. This will oblige us to compute the whole trajectory. The computation of the complete data set on Blagnac airport is ongoing.

Moreover, important variations of the CMC multipath pseudo-range error in static case have shown that the environment is still a source of uncertainties due to obstacles that are not taken into account (such as other aircraft).

\section{REFERENCES}

[Chen10] A. Chen. Development of a Hybrid Deterministic-Statistical GPS Multipath Simulator for Airport Navigation. PhD thesis, Université de Toulouse, 2010.

[Mon14] L. Montloin. GNSS Integrity Monitoring in the Presence of Singular Events. PhD thesis, Université de Toulouse, 2014. [Papou91] A. Papoulis, Probability, Random Variables and Stochastic Processes, $3{ }^{\text {rd }}$ edition, McGraw-Hill, 1991

[Boo00] Booth J., Murphy T., Clark B., Liu F, "Validation of the Airframe Multipath Error Allocation for Local Area Differential GPS", Proceedings of the IAN World Congress in association with the US ION ANNUAL MEETING, San Diego, CA, 26-28 June 2000

[Stein04] A. Steingass, A. Lehner, F. Pérez-Fontán, E. Kubista, M.J. Arbesser-Rastburg,” The high resolution aeronautical multipath navigation channel", IEEE PLANS, 2004

[Wan17] 'Group delay variations of GPS transmitting and receiving antennas', Lambert Wanninger, Hael Sumaya, Susanne Beer, Journal of Geodesy, September 2017, Volume 91, Issue 9, pp 1099-1116.

[ICAO05] ICAO. Aerodrome Design Manual - Part 2 - Taxiways, Aprons and Holding Bays - 4th edition. ICAO, 2005. 